\title{
So wirkt Homöopathie
}

\author{
Es sind die Umstände der homöo- \\ pathischen Sprechstunde, nicht aber \\ die Inhaltsstoffe homöopathischer \\ Präparate (die ohnehin nichts ent- \\ halten), die zu einer Besserung des \\ Befindens von Patienten mit aktiver, \\ aber stabiler rheumatoider Arthritis \\ beitragen.
}

—Zwischen 2006 und 2008 wurden Patienten mit seit mindestens zwei Jahren bestehender rheumatoider Arthritis, die eine mäßige Krankheitsaktivität, aber einen stabilen Verlauf aufwiesen (DAS $>2,6$ ), in eine fünfarmige placebokontrollierte Studie zur Wirksamkeit einer homöopathischen Behandlung einbezogen. Insgesamt konnten 83 Patienten aus drei Rheumambulanzen für die 24 Wochen dauernde Studie gewonnen werden, 56 Patienten beendeten die Studie wie vorgesehen.

Von den fünf Gruppen erhielten drei eine homöopathische Konsultation, zwei nicht. Die Patienten mit homöopathischer Behandlung wurden weiter randomisiert in eine Gruppe mit individualisierter Therapie, einer homöopathischen Komplextherapie für die rheumatoide Arthritis und Placebo. Die Gruppe ohne homöopathische Konsultation unterteilte man in eine mit homöopathischer Komplextherapie und ebenfalls eine Placebogruppe. Hier konnte keine dritte Gruppe gebildet werden, weil die individualisierte Therapie nur im Rahmen einer homöopathischen Konsultation durchgeführt werden kann.

Primäres Studienziel war es, den Anteil von Patienten zu finden, der eine 20\%ige Verbesserung anhand der ACRKriterien erlebte. Außerdem wurde der Verlauf des globalen Befindens anhand eines etablierten Scores festgestellt. Sekundäre Studienziele beinhalteten das Zählen von schmerzhaften und geschwollenen Gelenken (DAS-28), den Schweregrad der Erkrankung, der Schmerzen und der Entzündungsparameter. 77 Patienten konnten in die ITTAnalyse einbezogen werden.

\author{
Homöopathische \\ Globuli: Auf das Ritual, \\ nicht aber auf den \\ Inhalt kommt es an.
}

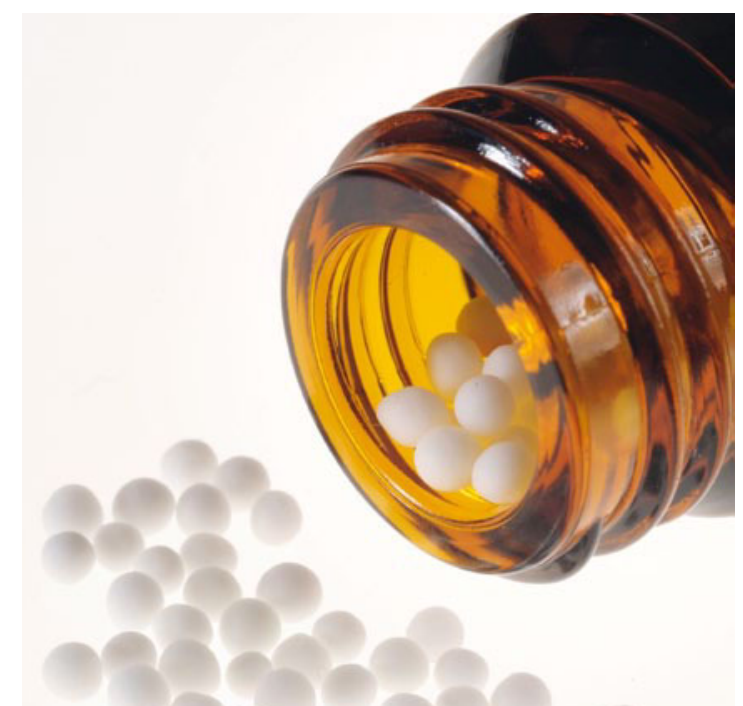

Bei den primären Studienzielen war in keiner der Gruppen ein signifikanter Unterschied zu beobachten. Allerdings hatten die Patienten, die in der homöopathischen Sprechstunde waren, eine statistisch signifikante Verbesserung bei den sekundären Studienzielen wie DAS-28, dem Schweregrad der Schmerzen und einer weniger negativen Einstellung zur Krankheit. Unabhängig von der sonstigen medikamentösen Behandlung führte die homöopathische Konsultation zu günstigen Effekten bei der Zahl der entzündeten und der geschwollenen Gelenke und zur Verbesserung des Gesamtzustandes.

\section{Kommentar}

Es handelt sich um die erste wissenschaftlich exakte Studie, die zeigt, dass der Nutzen einer homöopathischen Behandlung auf dem Konsultationsprozess und der Arzt-Patienten-Kommunikation beruht. Daher ist die Homöopathie auch bei vielen chronischen Erkrankungen wirksam, selbst wenn in den verabreichten Präparationen kein einziges Molekül der angeblichen Wirksubstanz mehr vorhanden ist. Die Verabreichung einer Substanz ist im Sinn einer spezifischen Wirkung auch gar nicht erforderlich, sondern allenfalls als - wenngleich durchaus wirksames - Ritual aufzufassen. Homöopathisch orientierte Ärzte haben wahrscheinlich ein besseres Kommunikationsverhalten, zeigen mehr Empathie und sind in der Lage, den Patienten neue Hoffnung schöpfen zu lassen. Er fühlt sich in ihren Händen als Individuum behandelt und persönlich angesprochen. Man darf gespannt sein, wie der manchmal mit religiöser Inbrunst geführte Streit zwischen Homöopathie und Schulmedizin durch diese Studie beeinflusst wird. Die Schulmediziner könnten sich am Verhalten der Homöopathen ihren Patienten gegenüber schon mal ein Beispiel nehmen.

H. S. FÜESSL 